\title{
A PROSPECTIVE STUDY OF DRUG-DRUG INTERACTIONS AND ADVERSE DRUG REACTIONS AMONG STROKE PATIENTS IN A TERTIARY CARE HOSPITAL
}

\author{
ATHIRA JITH, CHAITHANYA T KUMAR, JOFFEY SARA JOY, KAVITHA SAMY P*, SAMBATH KUMAR R
}

Department of Pharmacy Practice, J.K.K Nattraja College of Pharmacy, Kumarapalayam, Namakkal, Tamil Nadu - 638 183, India.

Email: kavitha.p119@gmail.com

Received: 23 June 2016, Revised and Accepted: 30 August 2016

\section{ABSTRACT}

Objective: Our study attempts to get an insight into the drug-drug interactions (DDIs) and adverse drug reactions (ADRs) among stroke patients from the Neurology Department in a private hospital.

Methods: In a prospective study spanning 6 months (May to October 2015), we have analyzed the prescription data of 221 patients with both ischemic and hemorrhagic stroke. Gender, age, social habits, length of stay, drug utilization pattern, DDIs reported from the database and clinically observed, and ADRs of individual drugs were observed among the patients. Of 221 cases, 208 (94.11\%) were ischemic, 12 (5.43\%) were hemorrhagic, and $1(0.45 \%)$ was transient ischemic attack.

Results: A number of 140 patients were males and 80 were females. The mean age of the patients was between 41 and 70 years. In ischemic patients, 357 major, 282 moderate, and 38 mild DDIs were reported using a specific database, while in hemorrhagic patients, 10 major, 7 moderate, and 1 mild interaction were reported using a specific database. 18 DDIs were observed clinically and confirmed with evidence. 8 DDI-induced ADRs and 10 ADRs caused by individual drugs were observed in our study population. 18 DDIs were observed clinically and confirmed with evidence. 8 DDI-induced ADRs and 10 ADRs caused by individual drugs were observed in our study population.

Conclusion: Drugs if wrongly prescribed may cause negative outcomes and pose significant challenge to health-care providers and may contribute to morbidity and mortality. Clinical pharmacist can play an important role in identifying and resolving drug-related problems through pharmaceutical care practices.

Keywords: Stroke, Drug-drug interactions, Adverse drug reactions, Clinical pharmacist.

(C) 2016 The Authors. Published by Innovare Academic Sciences Pvt Ltd. This is an open access article under the CC BY license (http://creativecommons org/licenses/by/4. 0/) DOI: http://dx.doi.org/10.22159/ajpcr.2016.v9s3.13651

\section{INTRODUCTION}

Stroke is a brain attack. It happens when the blood supply to part of the brain is cut off [1]. There are two main types of cerebrovascular accident, or stroke: An ischemic stroke is caused by a blockage and a hemorrhagic stroke is caused by a breakage in a blood vessel. In both cases, part of the brain is deprived of blood and oxygen, causing the brain cells to die [2]. Ischemic stroke constitutes about $85 \%$ and hemorrhagic stroke constitutes $15 \%$ of the total stroke occurrences [3]. Worldwide, cerebrovascular diseases are responsible for 6.15 million deaths [4]. Stroke is one of the leading causes of death and disability in India [5]. The estimated adjusted prevalence rate of stroke range is 84-262/100,000 in rural and 334-424/100,000 in urban areas [6]. High blood pressure (BP) is the most important risk factor for stroke, contributing to about $50 \%$ of all strokes $[7,8]$. One in five strokes is fatal. Stroke causes about $7 \%$ of deaths in men and $10 \%$ of deaths in women [9]. About $15 \%$ of strokes are hemorrhagic and $85 \%$ ischemic.

A drug interaction is a situation, in which a substance (usually another drug) affects the activity of a drug when both are administered together This action can be synergistic (when the drug's effect is increased) or antagonistic (when the drug's effect is decreased) or a new effect can be produced that neither produces on its own [10]. Medical complications are believed to be an important problem after acute stroke and present potential barriers to optimal recovery. For example, clopidogrel is a widely used medication for atherosclerotic diseases, particularly in the prevention of thrombotic events in stable cardiovascular disease, and as a secondary prevention after myocardial infarction and stroke.

DDI in patients receiving multiple drug therapy is a major concern. For example, anticoagulants and antiplatelet drugs (e.g., warfarin, clopidogrel, and acetylsalicylic acid) are key therapeutic agents in the treatment of cerebrovascular diseases. However, DDIs may lead to a greatly increased risk of gastrointestinal (GI) bleeding when these drugs are combined [4]. Patients with atherosclerotic disease are frequently treated for hypercholesterolemia with both clopidogrel and atorvastatin or another statin, and it was noted that the antiplatelet activity of clopidogrel was diminished significantly when patients were also taking atorvastatin [5]. The highly reported drug-related problem in stroke patients is drug interactions, followed by drug use without indication and adverse drug reactions (ADRs) [11].

ADR is defined as any noxious, unintended, and undesired effect of a drug, which occurs at doses used in human for prophylaxis, diagnosis, or therapy. Heightened interest of ADRs was stimulated by the thalidomide tragedy in the 1960s. ADRs may rank as sixth leading cause of death. In histochemical studies, the use of enalapril led to the development of numerous side effects, which commonly occur during the treatment. One of the most common side effects is an erythematous maculopapular skin rash, which occurs in up to $30 \%$ of patients treated with the drug and which seems to be related to dose [12]. Anticoagulant drugs are among the most common medications that cause adverse drug events in hospitalized patients. In our study also, anticoagulants constitute the prior class of drug used in the therapeutic management of stroke patients.

\section{METHODS}

Study site

The study was conducted in a private hospital in Erode.

\section{Study population}

The study included stroke patients undergone treatment. 
Study design

The design of the study was of prospective type.

\section{Period of the study}

The study was performed for 6 months (May-October 2015).

Inclusion criteria

- Both male and female patients

- Patients of all age groups

- Stroke patients with comorbidities.

\section{Exclusion criteria}

- Stroke patients with skin diseases

- Pregnant stroke patients and psychiatric patients.

\section{Data collection procedure}

During case collection, the study purpose was explained to the patients and bystanders verbally. The details of the stroke cases were entered into data entry forms. The identified DDIs were reported and documented in the corresponding DDI form. The identified ADRs were entered in the ADR form, and the causality assessment of ADRs was performed using Naranjo scale. The photographs of ADRs were taken with the consent of the patient and their bystanders.

\section{RESULTS AND DISCUSSION}

Our prospective study focused on the DDIs and ADRs among stroke patients and was carried out in a private hospital in Erode, Tamil Nadu, from May to October 2015.

A total of 221 patients were included in the study. Table 1 describes the demographic details of the study patients. Of the 221 patients, $141(63.80 \%)$ were male and $80(36.19 \%)$ were females. The occurrence of stroke was more in men than women because the secondary factors such as high BP and vasoconstriction were more common in men and on the other hand estrogen helps in the health of brain capillaries in women, thereby lowering the risk of stroke [13]. Patients from all the age groups were included in the study. The majority of the study population were adults since aging is a risk factor for the occurrence of stroke. From the data, non-vegetarians (85.52\%) were dominant than vegetarians $(14.47 \%)$ among the study population because the risk factors for stroke was more among them.

Smoking, alcoholism, and tobacco use were some root causes for the occurrence of stroke which was also proven in our study. Pandian and Sudhan conducted a study on stroke epidemiology and stroke care services in India, which coincides with the demographic data in our study [13].

Types of stroke found in 221 patients were found. Of the 221 patients, $12(5.43 \%)$ patients had hemorrhagic stroke, $208(94.11 \%)$ patients had ischemic stroke, and $1(0.45 \%)$ patient had transient ischemic attack. Sridharan et al. also conducted a study which states almost similar finding related to type of stroke as in our study [14]. The number and corresponding percentage of drug utilization pattern of drugs among stroke patients are presented (Fig. 1). From the data, antiulcer drugs, hypolipidemic drugs, and antihypertensive rate the higher utilization of drugs among the selected population. Of 208 patients, a total of $154(74.03 \%)$ patients consumed antiulcer drugs, 147 (70.67\%) consumed hypolipidemic drugs, 140 (67.30\%) antihypertensive, 106 (50.96\%) aspirin, and 139 (66.82\%) consumed clopidogrel. The concurrent use of many drugs or its co-administration with a length of stay of many days created medication errors in the stroke patients, which are depicted also in a study conducted by Subhash et al. [15]

From the collected data, about 14 ischemic stroke patients consumed 16-20 drugs and 52 patients consumed 11-15 drugs. 123 patients rated high for consuming 6-10 drugs in their prescription while only 9 and 10 patients consumed 0-5 and 20-25 number of drugs, respectively. In case of hemorrhagic stroke patients, also, the higher rate falls under
6-10 number of drugs which includes 4 patients. It was then followed by $6-10$ and $16-20$ categories with 2 patients each. 3 patients consumed 0-5 number of drugs and only one patient consumed 20-25 drugs. Polypharmacy was a major factor for the development of DDIs and ADRs which agreed with the study conducted by Sridharan et al. [14].

The DDIs according to the type of stroke are shown in Table 2. The DDIs were predominantly found among 677 (96.99\%) ischemic stroke patients. Anticoagulants, antiplatelets, statins, and antihypertensives were more administrated in ischemic stroke for the lysis of clot that occludes the cerebral artery which caused the major DDIs, while in hemorrhagic stroke, surgical interventions were mainly the primary step undertaken. $18(2.57 \%)$ interactions were found among hemorrhagic stroke patients and $3(0.443 \%)$ were found in transient ischemic attack. In India, ischemic stroke constitutes about $85 \%$ of stroke-associated disability and drug-related problems, followed by $15 \%$ of hemorrhagic stroke. This result agreed with the assessment of drug-related problems carried out among stroke patients by Celin et al. [10].

Classification of DDIs according to severity scale and type of interaction in ischemic stroke patients is presented in Table 3. On the basis of

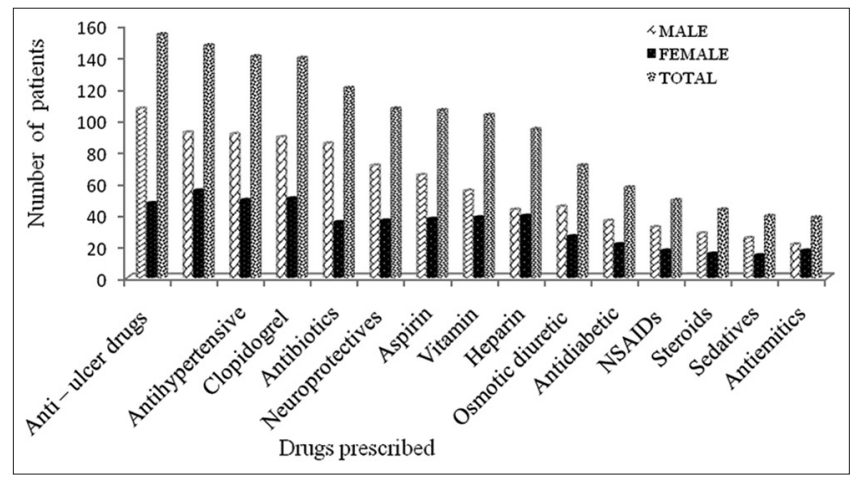

Fig. 1: Drug utilization pattern among the study population $(n=221)$

Table 1: Demographic profile of stroke patients $(n=221)$

\begin{tabular}{ll}
\hline Parameters & Number of patients (n=221) (\%) \\
\hline Gender & $141(63.80)$ \\
$\quad$ Male & $80(36.19)$ \\
Female & $13(5.88)$ \\
Age (years) & $175(79.18)$ \\
$0-40$ & $33(14.93)$ \\
$41-70$ & $32(14.47)$ \\
$71-90$ & $189(85.52)$ \\
Diet & $124(56.10)$ \\
$\quad$ Vegetarian & $118(53.39)$ \\
$\quad$ Non-vegetarian & $19(8.59)$ \\
Social habits & \\
Smoker & $42(19)$ \\
Alcoholic & $179(80.99)$ \\
$\quad$ Betel nut & \\
Length of stay (days) & \\
$\quad<10$ & \\
$>10$ &
\end{tabular}

Table 2: DDI classification according to type of stroke

\begin{tabular}{llll}
\hline Type of stroke & $\begin{array}{l}\text { Number } \\
\text { of cases }\end{array}$ & $\begin{array}{l}\text { Number of DDIs } \\
(\mathbf{n = 6 9 8 )} \text { (\%) }\end{array}$ & $\begin{array}{l}\text { \% of } \\
\text { interactions }\end{array}$ \\
\hline Ischemic stroke & 208 & 677 & 96.99 \\
Hemorrhagic stroke & 12 & 18 & 2.57 \\
Transient ischemic attack & 1 & 3 & 0.43 \\
\hline
\end{tabular}

DDIs: Drug-drug interactions 
severity scale of DDIs, the interactions were classified into severe 357 (52.73\%), moderate 282 (41.65\%), and mild 38 (5.61\%). It was due to the use of drugs such as anticoagulants, antiplatelets, statins, antihypertensive, and also drugs used for symptomatic treatment in stroke that led to major effects such as bleeding, GI tract (GIT) ulceration, QT prolongation, blunting of drug activity, and potent toxicities of certain drugs. On classification according to the type of interaction, 376 (56.45\%) pharmacodynamics and 290 (43.54\%) pharmacokinetic interactions were found.

Classification of DDIs according to severity scale and type of interaction in hemorrhagic stroke patients were also studied. On the basis of severity scale of DDIs, the interactions were classified into severe $10(55.5 \%)$, moderate 7 (38.88\%), and mild 1 (5.55\%). It was due to the use of drugs such as neuroprotectives, statins, osmotic diuretics, antihypertensive, and also drugs used for symptomatic treatment in stroke which would cause severe interactions. On classification according to the type of interaction, 10 (55.55\%) pharmacodynamic and $8(44.44 \%)$ pharmacokinetic interactions were found.

Clinically important severe, moderate, and mild DDIs among the prescribed drugs of ischemic stroke patients were identified using the database Micromedex, respectively. DDIs were found most frequently in patients who stayed in hospital for more than 5 days and mainly occurred between the drugs administered orally. The most common drug classes involved in DDIs were the antiplatelets, anticoagulants, antihypertensives, and statins. Among these, clopidogrel, aspirin, amlodipine, atorvastatin, furosemide, phenytoin, domperidone, heparin, and enalapril were the drugs which caused common clinically important drug interactions. These findings were supported by a study in a tertiary care hospital for DDIs among stroke patients conducted by Subash et al. [16].

Clinically important DDIs among the prescribed drugs of hemorrhagic stroke patients were also included in this study. DDIs were found more frequently in patients who stayed in hospital for more number of days. DDIs were lesser in hemorrhagic stroke patients as the number of drugs was lesser in them as compared to ischemic stroke patients. The most common drug classes involved in DDIs were the antiplatelets, anticoagulants, antihypertensives, and statins. Among these, clopidogrel, aspirin, amlodipine, atorvastatin, furosemide, phenytoin, domperidone, heparin, and enalapril were the drugs which caused common clinically important drug interactions. These findings

Table 3: Classification of DDI based on the severity scale and type of interactions in ischemic stroke patients

\begin{tabular}{ll}
\hline & Number of interactions (\%) \\
\hline Severity & $357(52.73)$ \\
Severe & $282(41.65)$ \\
Moderate & $38(5.61)$ \\
Mild & \\
Type & $290(43.54)$ \\
Pharmacokinetic & $376(56.45)$ \\
Pharmacodynamic &
\end{tabular}

DDIs: Drug-drug interactions were supported by a study in a tertiary care hospital for DDIs among stroke patients conducted by Subash et al. [16].

Clinically observed and confirmed DDIs among stroke patients in our study are presented in Table 4 . The concurrent use of tablet aspirin and tablet diclofenac for more than 5 days had led to GI bleeding in a female patient with blood stains in stool. The mechanism that caused this bleeding may be mainly the back diffusion of $\mathrm{H}^{+}$ions across the gastric barrier with physical erosion, prolonged platelet bleeding, and the effect of low $\mathrm{pH}$ [17]. The physician discontinued the drug diclofenac and the patient was relieved from the effect.

Thrombocytopenia as a serious ADR has been identified in 2 patients who took tablet clopidogrel and tablet diclofenac concurrently. The ADR was confirmed using the hematological studies of the patients, which was also evident from the study conducted by Eric et al. in 2010 [18].

Swollen gums had been observed as a moderate ADR in 1 patient. In our observation, it had not changed to hyperplasia only swelling was there caused by the drug phenytoin when it has been concurrently taken with clopidogrel which may have increased the phenytoin plasma concentration and led to this moderate interaction [19].

Concurrent administration of tablet enalapril and tablet spironolactone had caused postural hypertension in 3 patients. This effect was identified by better interaction with the patients.

Hypokalemia had occurred in 3 patients who took tablet spironolactone and tablet aspirin which was evident from the patient's lab report and also in 2 patients injection $\mathrm{KCl}$ had been administered to maintain the serum potassium level.

Tablet escitalopram was the main antidepressant drug used in our study population and tablet domperidone as the antiemetic drug. The concurrent use of these two drugs had caused abnormal electrocardiogram in patients who do not have a previous history of cardiac disease. QT wave prolongation was observed in patients.

A minor interactive effect, but a common interaction was found among 4 patients, is that the persisted constipation even after the use of tablet bisacodyl. This effect was occurred due to the concurrent administration of the drugs ranitidine and bisacodyl. This was identified as an administrative error by the staff who failed to give ranitidine at least 30 minutes before food, and thus, the drug may have caused reduced absorption of bisacodyl and led to subtherapeutic effect.

Clinically observed DDI-induced ADRs are illustrated in Table 5. In two patients, the concurrent use of tablet clopidogrel and tablet phenytoin caused muscle stiffness (mild ataxia) as the ADR. This may be due to the increased phenytoin concentration in plasma precipitated by clopidogrel by metabolic induction [20].

The concurrent use of tablet amlodipine and tablet atenolol resulted in hypotension in 1 patient, which was life-threatening (90/65 $\mathrm{mm} \mathrm{Hg}$ ). The patient was reversed into the stable BP level by injecting

Table 4: Clinically observed and confirmed DDIs among stroke patients

\begin{tabular}{|c|c|c|c|c|c|}
\hline Interacting drugs & Observed interaction effect & Severity & Type & $\begin{array}{l}\text { Number of } \\
\text { patients }(n=221)\end{array}$ & $\begin{array}{l}\text { Number of patients taking } \\
\text { this combination }(n=221)\end{array}$ \\
\hline Tablet aspirin+Tablet amlodipine & GI bleeding & Severe & PD & 1 & 199 \\
\hline Tablet clopidogrel+Tablet diclofenac & Thrombocytopenia & Severe & PD & 2 & 113 \\
\hline Tablet clopidogrel+Tablet phenytoin & Swollen gums & Moderate & PK & 1 & 98 \\
\hline Tablet enalapril+Tablet furosemide & Postural hypotension & Moderate & PD & 3 & 102 \\
\hline Tablet spironolactone+Tablet aspirin & Hypokalemia & Moderate & PD & 3 & 31 \\
\hline Tablet domperidone+Tablet escitalopram & QT Wave prolongation-abnormal ECG & Major & PK & 4 & 132 \\
\hline
\end{tabular}

DDIs: Drug-drug interactions, ECG: Electrocardiogram, GI: Gastrointestinal 
sympathomimetic. Instead of tablet atenolol $50 \mathrm{mg}$, physician could have reduced the dose to $25 \mathrm{mg}$.

High BP was observed in 2 patients when tablet aspirin and tablet atenolol were concurrently administered.

Concurrent administration of tablet aspirin and tablet diclofenac resulted in GI ulceration. This was evident with the impression of abdominal ultrasonography of the patient which depicted ulceration [21].

Pedal edema persisted in 2 patients when tablet furosemide and tablet diclofenac were concurrently given. The co-administration of these two drugs resulted in the blunting of the diuretic effect of the furosemide and reduced antihypertensive effects [20]

Stroke therapy and the ADRs developed in the study population were discussed in this study (Table 6). According to prescription, 171 patients were taking amlodipine making it the most utilized drug in the current study patients, followed by atorvastatin 95 in the context of ADRs. Phenytoin was prescribed in 44 patients, enalapril in 47 patients, and citalopram in 52 patients.

Rhabdomyolysis was developed as an ADR of atorvastatin in one patient who had a history of old ischemic stroke and past drug history of atorvastatin for 10 years. This prolonged use of the drug may have increased the production or use of ATP by skeletal muscle which impaired or increased energy within the muscles and led to increased pressure and caused rhabdomyolysis [22]

Amlodipine caused pedal edema in 3 patients. Among them, 1 was recovered fast within 3 days due to the dosage adjustment in the drug. Pedal edema mainly occurred in amlodipine users which may be due to the arteriolar dilation, thus causing flooding of venules and leakage of fluids into interstitial fluids [23]

One patient developed the ADR nystagmus after the IV administration of phenytoin $200 \mathrm{mg}$ BID. Phenytoin has a narrow therapeutic range only; hence, here, the half-life of the drug may have increased with the drug on higher concentration which may have led to increased plasma levels and caused toxicity [19].

Escitalopram developed right lower limb edema in a female patient admitted with ischemic stroke together with swelling of palms and left lower limb edema in another male patient. It may be due to the strong serotonergic effect of escitalopram which caused vascular hyperpermeability [24].

Skin rash with itching was found in a patient taking enalapril BID and the reaction was resolved after the withdrawal of drug. Skin rash may have occurred due to the hypersensitivity type B reaction [12].

Atorvastatin developed dreadful constipation in a female patient with pain during the passage of bowel movements which was reduced after taking laxative syrup. The underlying mechanism may be the atorvastatin-induced muscle weakness and reduced peristalsis by the lactone metabolite of the drug which caused mitochondrial dysfunction.

Urethral bleeding occurred as a serious adverse effect of aspirin in a female patient taking $325 \mathrm{mg}$ of aspirin. The patient had compliance of blood stains in urine (hematuria) after $5^{\text {th }}$ day of admission in hospital which was confirmed by the urine analysis showing red blood cells in urine. This may be due to the reduced PG synthesis which had led to vasoconstriction at the glomerular level [25].

Table 7 depicts the system-wise ADR distribution. The organ systems involved in the ADRs were central nervous system, dermatologic, musculoskeletal system, GIT, and renal system. The organ system predominantly affected by the ADRs was musculoskeletal system. The drugs used in stroke patients caused limb edema, pedal edema, rhabdomyolysis, GIT bleeding, nystagmus, skin rashes, and constipation in patients as ADR

Based on dermatologic system, 1 patient was presented with skin rash due to enalapril.

In the musculoskeletal system, pedal edema was observed in 3 patients who took Amlodipine and limb edema in 2 patients who took escitalopram

In GIT constipation and in renal system urethral bleeding were observed as the ADRs.

Rhabdomyolysis was found as a serious ADR in 1 patient taking atorvastatin [26]. A predominance of male patients (141) over females (80) was observed in the study. However, number of ADR was high in females (6 of 10).

Table 5: Observed DDI-induced ADR among stroke patients

\begin{tabular}{|c|c|c|c|}
\hline Interacting drugs & DDI-induced ADR & Number of patients & $\begin{array}{l}\text { Number of patients taking } \\
\text { this combination }(n=221)\end{array}$ \\
\hline $\begin{array}{l}\text { Tablet clopidogrel+Tablet } \\
\text { phenytoin }\end{array}$ & Muscle stiffness & 2 & 98 \\
\hline Tablet atenolol+Tablet amlodipine & Hypotension & 1 & 107 \\
\hline Tablet aspirin+Tablet atenolol & Hypertension & 2 & 120 \\
\hline Tablet aspirin+Tablet diclofenac & GI ulceration & 1 & 114 \\
\hline Tablet furosemide+Tablet diclofenac & Pedal edema persists & 2 & 121 \\
\hline
\end{tabular}

DDI: Drug-drug interaction, ADR: Adverse drug reactions

Table 6: Status of ADR developed in study population

\begin{tabular}{lllll}
\hline Drugs & $\begin{array}{l}\text { Number of patients } \\
\text { taking the drug }\end{array}$ & $\begin{array}{l}\text { Number of patients } \\
\text { presenting ADR (\%) }\end{array}$ & $\begin{array}{l}\text { ADR occurrence } \\
\text { (n=221) (\%) }\end{array}$ \\
\hline Amlodipine & 171 & $3(1.7)$ & 1.3 & Pype of reactions observed \\
Phenytoin & 44 & $1(2.2)$ & 0.44 & Nystagmus \\
Enalapril & 47 & $1(2.1)$ & 0.45 & Skin rash \\
Atorvastatin & 95 & $1(1.05)$ & 0.45 & Rhabdomyolysis \\
Escitalopram & 52 & $2(3.8)$ & 0.9 & Limb oedema and swelling of palms \\
Aspirin & 197 & $1(0.5)$ & 0.45 & Urethral bleeding \\
Atorvastatin & 95 & $1(1.05)$ & 0.45 & Constipation with pain \\
\hline
\end{tabular}

ADR: Adverse drug reactions 
Table 7: System-wise ADR distribution

\begin{tabular}{|c|c|c|c|c|c|c|c|}
\hline Organ system & Type of reaction & PHYN & AMLO & ENPL & ESPM & ATOR & ASP \\
\hline CNS & Nystagmus & + & - & - & - & - & - \\
\hline Dermatologic & Skin rashes & - & - & + & - & - & - \\
\hline \multirow[t]{3}{*}{ Musculoskeletal } & Limb edema & - & - & - & + & - & - \\
\hline & Pedal edema & - & + & - & - & - & - \\
\hline & Rhabdomyolysis & - & - & - & - & + & - \\
\hline GIT & Constipation & - & - & - & - & + & - \\
\hline Renal & Urethral bleeding & - & - & - & - & - & + \\
\hline
\end{tabular}

ADR: Adverse drug reactions, PHYN: Phenytoin, AMLO: Amlodipine, ENPL: Enalapril, ESPM: Escitalopram, ATOR: Atorvastatin, ASP: Aspirin, GI: Gastrointestinal tract, CNS: Central nervous system, +: Positive, -: Negative

Table 8: Causality assessment of observed ADRs

\begin{tabular}{lll}
\hline ADR scale category & Score & Number of cases \\
\hline Definite & $>9$ & - \\
Probable & $5-8$ & 09 \\
Possible & $1-4$ & 01 \\
Doubtful & 0 & - \\
\hline
\end{tabular}

ADR: Adverse drug reactions

In age-wise ADR distribution, patients of 41-70 years age range were more in the study (175 of 221). A number of ADRs were high (8 of 10) in adult stroke patients (41-70 years) than that of geriatric stroke patients.

The causality assessment of ADR with the drug is shown in Table 8. The causality assessment of ADRs using Naranjo's ADR assessment scale revealed that 9 cases of ADR had probable relationship with the drug while 1 case of ADR had possible relationship with the drug.

\section{CONCLUSION}

Stroke is a leading cause of death and disability worldwide. Therefore, stroke prevention is of great importance for public health. Our study highlighted the DDIs which was high amount in stroke patients prescribed with drugs in a tertiary care teaching hospital. DDI in patients receiving multiple drug therapy is a major concern as such interaction may lead to increased risk of hospitalization and higher health-care cost. In our study, DDI mainly occurred between antihypertensive, anticoagulants, and antiplatelet. The significant proportions of patients with DDIs were occupied by males followed by females. In our study, we also depicted the ADR occurred among stroke patients. Antihypertensive contributed much to the occurrence of ADRs. Further research studies are needed to investigate the DDIs and ADRs among stroke patients in future. Drugs if wrongly prescribed may cause negative outcomes and pose a significant challenge to healthcare providers and may contribute to morbidity, mortality and lead to a negative quality of life in patients. The clinical pharmacist can play an important role in identifying and resolving DDI and ADR through absolute pharmaceutical care practices.

\section{REFERENCES}

1. Sacco RL, Kasner SE, Broderick JP, Caplan LR, Connors JJ, Culebras A, et al. An updated definition of stroke for the $21^{\text {st }}$ century: A statement for healthcare professionals from the American heart association/ American stroke association. Stroke 2013;44(7):2064-89.

2. Albers GW, Caplan LR, Easton JD, Fayad PB, Mohr JP, Saver JL, et al. Transient ischemic attack - proposal for a new definition. N Engl J Med 2002;347(21):1713-6.

3. Sacco RL, Kargman DE, Gu Q, Zamanillo MC. Race-ethnicity and determinants of intracranial atherosclerotic cerebral infarction. The Northern Manhattan Stroke Study. Stroke 1995;26:14-20.

4. WHO: The top 10 causes of death online address; 2011. Available from: http://www.who.int/mediacentre/factsheets/fs310/en/index.html. [Last accessed on $2015 \mathrm{Jul} 01]$.
5. Strong K, Mathers C, Bonita R. Preventing stroke: Saving lives around the world. Lancet Neurol 2007;6(2):182-7.

6. Lawes CM, Vander Hoorn S, Rodgers A; International Society of Hypertension. Global burden of blood-pressure-related disease, 2001. Lancet 2008;371:1513-8.

7. Townsend N, Wickramasinghe K, Bhatnagar P, Smolina K, Nichols M, Leal J, et al. Coronary Heart Disease Statistics. London: British Heart Foundation; 2012.

8. Katzan IL, Hammer MD, Hixson ED, Furlan AJ, Abou-Chebl A, Nadzam DM; Cleveland clinic health system stroke quality improvement team. Utilization of intravenous tissue plasminogen activator for acute ischemic stroke. Arch Neurol 2004;61(3):346-50.

9. Pandian JD, Srikanth V, Read SJ, Thrift AG. Poverty and stroke in India: A time to act. Stroke 2007;38(11):3063-9.

10. Celin AT, Seuma J, Ramesh A. Assessment of drug related problems in stroke patients admitted to a south Indian tertiary care teaching hospital IJOPP 2012;5(4):28-33.

11. Feigin VL, Lawes CM, Bennett DA, Barker-Collo SL, Parag V. Worldwide stroke incidence and early case fatality reported in 56 population-based studies: A systematic review. Lancet Neurol 2009;8(4):355-69.

12. Furness PN, Goodfield MJ, MacLennan KA, Stevens A, Millard LG Severe cutaneous reactions to captopril and enalapril; histological study and comparison with early mycosis fungoides. J Clin Pathol 1986;39(8):902-7.

13. Pandian JD, Sudhan P. Stroke epidemiology and stroke care services in India. J Stroke 2013;15(3):128-34

14. Sridharan SE, Unnikrishnan JP, Sukumaran S, Sylaja PN, Nayak SD, Sarma PS, et al. Incidence, types, risk factors, and outcome of stroke in a developing country: The Trivandrum Stroke Registry. Stroke 2009;40(4):1212-8

15. Subhash V, Damodar G, Ravikanth S, Vijayakumar G. Drug utilisation pattern of stroke patients in a tertiary care hospital. IJPCR 2012;2(1):3-7.

16. Subash V, Ravikanth S, Ayesha S, Dheeraj K. Drug-drug interaction occurring during hospital stay among stroke patients. Afr J Pharm Pharmacol 2012;37(6):2670-4.

17. Mehul D, Thuy D, Rebecca K, Naznin D. Significant acute kidney injury due to non - steroidal anti- inflammatory drugs: Inpatient setting. Pharmceuticals 2010;3(4):1279-85.

18. Eric R, Wei C, Dominick JA. Clopidogrel - drug interactions. J Am Coll Cardiol 2011;57(11):1151-63.

19. Vineetha BM, Justin K, Madhan R, Hathur BG. Phenytoin toxicity: A case report. J Young Pharm 2015;3(7):22-7.

20. Piazza G, Nguyen TN, Cios D, Labreche M, Hohlfelder B, Fanikos J, et al. Anticoagulation-associated adverse drug events. Am J Med 2011;124(12):1136-42.

21. Gartner AH. Aspirin-induced gastritis and gastrointestinal bleeding. J Am Dent Assoc 1976;93(1):111-7.

22. Supsongserm P, Boonmuang P, Nathisuwan S, Chaiyakunapruk KT. Risk factors related to rhabdomyolysis in Thai statin users: A casecontrol study. MUJPS 2013;40(4):1-7.

23. Aman G. Evaluation of amlodipine induced pedal edema (Eape) study. J Pharmacovigilance 2014;2:5.

24. Ravi PB, Ravishankar GM, Andrade C. Bilateral peripheral edema as a rare adverse effect of escitalopram. Indian J Psychiatry 2014;56(1):97.

25. Delaney JA, Opatrny L, Brophy JM, Suissa S. Drug drug interactions between antithrombotic medications and the risk of gastrointestinal bleeding. CMAJ 2007 14;177(4):347-51.

26. Omar MA, Wilson JP. FDA adverse event reports on statin-associated rhabdomyolysis. Ann Pharmacother 2002;36(2):288-95. 